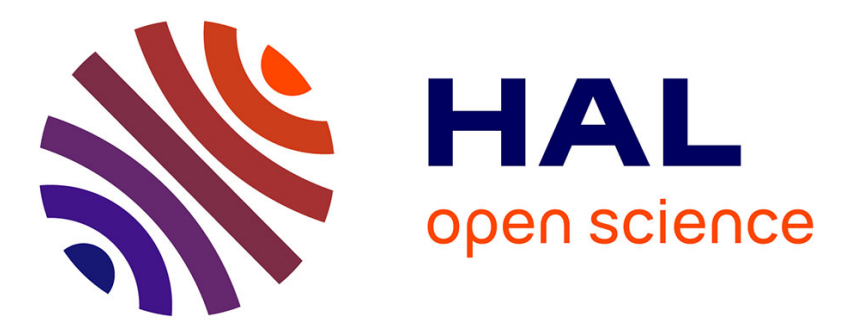

\title{
Characterization of hysteretic stress-strain behavior using the integrated Preisach density
}

\author{
V. Aleshin, W. Desadeleer, M. Wevers, K. van den Abeele
}

\section{To cite this version:}

V. Aleshin, W. Desadeleer, M. Wevers, K. van den Abeele. Characterization of hysteretic stress-strain behavior using the integrated Preisach density. International Journal of Non-Linear Mechanics, 2008, 43 (3), pp.151. 10.1016/j.ijnonlinmec.2007.12.009 . hal-00501769

\section{HAL Id: hal-00501769 \\ https://hal.science/hal-00501769}

Submitted on 12 Jul 2010

HAL is a multi-disciplinary open access archive for the deposit and dissemination of scientific research documents, whether they are published or not. The documents may come from teaching and research institutions in France or abroad, or from public or private research centers.
L'archive ouverte pluridisciplinaire HAL, est destinée au dépôt et à la diffusion de documents scientifiques de niveau recherche, publiés ou non, émanant des établissements d'enseignement et de recherche français ou étrangers, des laboratoires publics ou privés. 


\section{Author's Accepted Manuscript}

-

NON-LINEAR

MECHANICS

Characterization of hysteretic stress-strain behavior using the integrated Preisach density

V. Aleshin, W. Desadeleer, M. Wevers, K. Van Den Abeele

PII: S0020-7462(07)00232-6

DOI: doi:10.1016/j.ijnonlinmec.2007.12.009

Reference: NLM 1426

To appear in: International Journal of NonLinear Mechanics

Received date: 25 May 2007

Revised date: 3 November 2007

Accepted date: 18 December 2007

Cite this article as: V. Aleshin, W. Desadeleer, M. Wevers and K. Van Den Abeele, Characterization of hysteretic stress-strain behavior using the integrated Preisach density, International Journal of Non-Linear Mechanics (2007), doi:10.1016/j.ijnonlinmec.2007.12.009

This is a PDF file of an unedited manuscript that has been accepted for publication. As a service to our customers we are providing this early version of the manuscript. The manuscript will undergo copyediting, typesetting, and review of the resulting galley proof before it is published in its final citable form. Please note that during the production process errors may be discovered which could affect the content, and all legal disclaimers that apply to the journal pertain. 


\title{
Characterization of hysteretic stress-strain behavior using the integrated Preisach density
}

\author{
V. Aleshin ${ }^{1}$, W. Desadeleer ${ }^{1}$, M. Wevers ${ }^{2}$, and K. Van Den Abeele ${ }^{1}$
}

${ }^{1}$ K.U.Leuven Campus Kortrijk, Interdisciplinary Research Center, E. Sabbelaan, 53, B-8500 Kortrijk, Belgium

${ }^{2}$ K.U.Leuven, Dept. of Materials Engineering, Kasteelpark Arenberg, 44

B-3001 Heverlee, Belgium

koen.vandenabeele@kuleuven-kortrijk.be

(received 


\section{Abstract}

In this paper, we introduce the concept of Integrated Preisach-Mayergoyz (IPM) density to analyze static uniaxial compression tests at values well below the critical strength, and to characterize the elasticity of materials with hysteresis in their stress-strain relationship. The IPM density can be deduced from a particular force protocol following basic data treatment. The advantage of the IPM density over prior approaches is that no second order differentiation of the data is required which reduces the errors and uncertainties typical for past practice in the specific context of rock elasticity using scanning curves and PM density analysis. The characterization of the elasticity of the material is established in terms of a non-hysteretic strain contribution in the form of a nonlinear but reversible equation of state, and a hysteretic contribution represented by the IPM density. The IPM inversion procedure is tested for simulated stress-strain data subjected to additive noise, and the results are compared to the traditional methodology. In addition, we analyze the hysteretic and non-hysteretic characteristics of five natural building stones, and show evidence for a classification based on the inferred properties.

Keywords: hysteresis, rock mechanics, non-destructive testing, nonlinear constitutive behavior 


\section{Introduction}

The class of materials for which hysteresis is believed to be responsible for the nonlinear elastic and acoustical properties, is quite extensive (rocks, minerals, construction materials, composites, ceramics, polycrystalline metals, martensites). An enlightening exemplar for observing the separation of loading and unloading curves in static stress-strain tests is the family of sandstones, where cyclic static tests under pressures of a few tens of MPa's reveal a difference between the ascending and descending branches (loop thickness) which is comparable to the elastic strain itself. A similar branch separation can be observed in the stress-strain behavior of fatigued metals. This makes hysteresis a material state property of primary importance and, consequently, the problem of its characterization and its inversion from measurements arises.

A traditional model for the description of rate-independent hysteresis is the Preisach space approach [1] which was initially developed for ferromagnetics. Later, this methodology was generalized in the perspective of control theory, including the proofs of several important theorems by Krasnosel'skii and Pokrovskii [2], whose results are discussed in a classical paper by Mayergoyz [3]. In the 1990's, this formalism was applied for the elasticity of martensites [4] and geomaterials [5], and in this context the term PM model or PKM model (or space) was adopted. Key player in this model is the PM-density distribution which represents the statistical distribution of microscopic units with a bistable state within a representative volume.

As far as material characterization is concerned, the basic methodology for inverting the hysteretic material characteristic (i.e. the PM density) from experimentally obtained stress-strain data was published by Guyer et al. [6]. It was noted that the PMdensity inversion is a highly underdefined problem: the number of constraints that can 
be obtained from a few hysteretic loops is typically much smaller than the number of points defining the PM density (2D surface). To avoid this difficulty, one generally uses regularization (or "smoothening") methods (see for instance Tikhonov and Arsenin [7] for a fundamental description of the underlying theory). Guyer et al. [6] proposed three different ways to realize the regularization, resulting in three inversion algorithms: "simulated annealing" (direct minimization of a regularized objective function), "normal modes" (approximation of the PM density with an orthonormal set of functions) and "exponential decay" (imposing an explicit form of the PM density characterized by a limited number of model parameters). Visually, the agreement between the three obtained density surfaces from the same set of data is acceptable; however, an extensive quantitative comparison and error calculation has not been performed. Nonetheless, using numerical tests with simulated data, it is straightforward to calculate the actual PM density reconstruction error for any of these approaches directly.

In this paper, we look at the problem from a slightly different point. On one hand, the fact that the PM density inversion is underdefined can be compensated to a certain extent by operating a special stress protocol that consists of an extended sequence of loops with different upper and lower stresses. Even though the finite measurement precision limits the amount of experimental stress-strain data points, it can be significantly increased. On the other hand, we note that the problem of reconstructing the PM density is highly ill-posed: similar stress-strain responses may correspond to highly different PM distributions. Indeed, as shown by Mayergoyz [3], the PM density can be obtained as the second derivative of the hysteresis information contained in the measured data. However, it is well known that each differentiation procedure is an extremely bad conditioned operation, especially on noisy data, and tends to blow up 
small errors on the measurements. Furthermore, once the PM density has been inferred from the data by double differentiation, it is then necessary to perform a $2 \mathrm{D}$ integral calculation of the PM density over a specific area to predict the stress-strain curves in response to an arbitrary stress protocol.

The above mentioned traditional practice opens a simple question: is it possible to avoid the double differentiation and the subsequent double integration, and characterize the material by a property that relates to the data more directly? In other words, can we use for instance an integral characteristic instead of a differential one?

Sure enough, there is not much scientific progress in using an integral property instead of a differential one. However, from a practical point, the approach that will be outlined in the next paragraphs completely avoids solving an ill-posed problem and performing a badly conditioned operation. Indeed, we will show that the characterization of both hysteretic and non-hysteretic material elasticity can be solved with a precision of a few percents even for very noisy data, if we are not obliged to infer the traditional representation of the PM density.

Ideally, when solving the hysteretic elasticity characterization problem, the inferred material property describing the mechanical hysteresis should

not depend on the experimental conditions;

be able to predict results for new experiments;

and be obtained by means of a well-posed inversion.

Such a method is proposed in the present communication and is discussed in detail below for the treatment of simulated noisy data and real experimental data. 


\section{PM density and IPM density}

The traditional treatment of hysteresis using the Preisach space (or PM space) consists in the evaluation of the following expression for the uniaxial $\operatorname{stress}(\sigma)-\operatorname{strain}(\varepsilon)$ relationship:

$$
\varepsilon=\varepsilon_{N H}(\sigma)+\varepsilon_{H}(\sigma, \text { history of } \sigma)
$$

Here, $\varepsilon_{N H}(\sigma)$ is a one-variable function representing the non-hysteretic contribution (sometimes referred to as classical nonlinearity since it is generally approximated by a simple polynomial expansion [8]). The second term accounts for the hysteresis itself and is history-dependent. The dependence on the stress history appears in the following way. We suppose that any representative (physically small) volume of the material contains a large number of hysteretic mechanical units, which can be found in one of two states: "open" or "closed". A single mechanical element is assumed to be characterized by two stress values $\sigma_{o}$ and $\sigma_{c}$, so that it opens if $\sigma<\sigma_{o}$ when the actual stress $\sigma$ decreases, closes if $\sigma>\sigma_{c}$ when the stress $\sigma$ increases. In all other cases it keeps its previous strain state. Here we adopt the conventions (convenient for compression tests) that $\sigma$ and $\varepsilon$ are both positive for compression. Even though there is no experimental evidence for this, we can associate the hysteretic elements hypothetically with internal cracks, which are closed at higher compression and open at smaller values. It is then obvious that $\sigma_{o}<\sigma_{c}$ for any element. The conjectural association of hysteretic units with microcracks and partially contacting surfaces has been studied in recent work by Pecorari [9-10], and Aleshin and Van Den Abeele [11- 
13]. Experimental evidence for the connection between crack density and macroscopic effects of hysteresis can be found in Van Den Abeele et al. [14].

We assume that the material (or better, each representative volume) is characterized by a distribution $\rho\left(\sigma_{c}, \sigma_{o}\right)$ of hysteretic elements (called PM density) with characteristic stress values $\sigma_{o}$ and $\sigma_{c}$, so that $\rho\left(\sigma_{c}, \sigma_{o}\right) d \sigma_{c} d \sigma_{o}$ is the probability to find an element in the rectangle $\left(\sigma_{c}, \sigma_{c}+d \sigma_{c}\right) \times\left(\sigma_{o}, \sigma_{o}+d \sigma_{o}\right)$ and $\int_{-\infty}^{+\infty} \int_{-\infty}^{+\infty} \rho\left(\sigma_{c}, \sigma_{o}\right) d \sigma_{c} d \sigma_{o}=1 . \mathrm{We}$ also accept that open units do not contribute to the stress-strain relationship given in Eq. (1) and that the total hysteretic strain contribution is given by $\gamma$ when all elements are closed. Under these assumptions, the hysteretic addition $\varepsilon_{H}$ to the total strain simply equals the fraction of closed elements multiplied by $\gamma$.

$$
\varepsilon_{H}=\gamma \iint_{\Omega_{c}} \rho\left(\sigma_{c}, \sigma_{o}\right) d \sigma_{c} d \sigma_{o}=\iint_{\Omega_{c}} \tilde{\rho}\left(\sigma_{c}, \sigma_{o}\right) d \sigma_{c} d \sigma_{o}
$$

where $\Omega_{c}$ denotes the area in the $\left(\sigma_{c}, \sigma_{o}\right)$ - or PM-space containing the closed elements, and $\tilde{\rho}=\gamma \rho$. An example is displayed in Figure 1 in which the population of closed units is represented by the dark gray area. Taking into account the above accepted rules for individual element switching, any stress loading history leads to a configuration in the PM space in which the closed $\left(\Omega_{c}\right)$ and open $\left(\Omega_{o}\right)$ areas are divided by a staircase line (Figure 1) defined by the loading history and, in particular, carrying information about the relevant stress extrema (e.g. Krasnosel'skii and Pokrovskii [2].

Starting from the classical representation of the PM-density, $\rho\left(\sigma_{c}, \sigma_{o}\right)$, we can also define the integral PM density (IPM density) $H\left(\sigma_{c}, \sigma_{o}\right)$, which is a dimensionless quantity: 


$$
H\left(\sigma_{c}, \sigma_{o}\right)=\gamma \int_{\sigma_{o}}^{\sigma_{c}} d x \int_{\sigma_{o}}^{x} \rho(x, y) d y=\int_{\sigma_{o}}^{\sigma_{c}} d x \int_{\sigma_{o}}^{x} \tilde{\rho}(x, y) d y .
$$

The area of integration is a right-angled triangle defined by the corners $\left(\sigma_{c}, \sigma_{c}\right),\left(\sigma_{o}, \sigma_{o}\right)$ and $\left(\sigma_{c}, \sigma_{o}\right)$ (as for instance indicated in Figure 4 which will be discussed later). It is easy to prove that Eq. (2) can be rewritten as

$$
\varepsilon_{H}=H_{1}-H_{2}+H_{3}-\ldots \pm H_{n}=-\sum_{i=1, n}(-1)^{i} H_{i}
$$

where the values $H_{i}$ correspond to the IPM densities at the $\left(\sigma_{c}, \sigma_{o}\right)$ points $i=1,2 . . n$ on the staircase-line as illustrated in Figure 1. The last sign is " + " if $n$ is odd and "-" otherwise. Here, we assume for simplicity that the section 1-2 is vertical (in practice, this is easily achievable by introducing an additional point very closely to the first one). Otherwise, Eq. (4) should read: $\varepsilon_{H}=H_{2}-H_{3}+H_{4}-\ldots$.

The relation between the PM density and the IPM density can also be rewritten as follows:

$$
\tilde{\rho}\left(\sigma_{c}, \sigma_{o}\right)=-\frac{\partial^{2} H\left(\sigma_{c}, \sigma_{o}\right)}{\partial \sigma_{c} \partial \sigma_{o}} .
$$

As it will be shown later, the advantage of the IPM density is that its distribution can be found directly from the stress-strain data. In addition, we avoid the ill-posed operation of calculating the second derivative of potentially noisy data. As a result, the 
IPM density will appear to be a more relevant material characteristic than the conventional differential one.

\section{Data treatment procedure for IPM density reconstruction}

In this Section we formulate the essential algorithm for retrieving the IPM density from obtained stress-strain data. The proposed method requires a specific stress protocol as displayed in Figure 2. This protocol contains $N_{L}=N(N+1) / 2$ loading-unloading cycles, with $N$ an integer number (e.g. $N=8$ in Figure 2), and is constructed in such a way that each compression-decompression cycle in the left hand side of Figure 2 corresponds to one of the possible triangles in the PM space, formed by any two orthogonal grid lines in the figure on the right hand side. Inversely, each of the $N_{L}=N(N+1) / 2$ triangles that can be found on the $N \times N$ grid, corresponds to a compression-decompression cycle in the stress protocol (a single loop in the stress-strain relationship). In the rest of this paper, we will refer to this particular protocol as the $N$-level stress protocol.

Starting from the basic equations of the PM model, i.e. Eqs. (1-2), it is easy to verify that the thickness $\Delta \varepsilon$ of a hysteretic loop at a constant stress value $\sigma$ equals the integral of the PM density over a rectangle with corners $(\sigma, \sigma),\left(\sigma_{\max }, \sigma\right),\left(\sigma_{\max }, \sigma_{\min }\right)$ and $\left(\sigma, \sigma_{\min }\right)$, where $\sigma_{\min }$ and $\sigma_{\max }$ denote the lowest and highest stresses for this particular loop (as shown in Figure 3):

$$
\Delta \varepsilon=\left|\varepsilon^{\uparrow}(\sigma)-\varepsilon^{\downarrow}(\sigma)\right|=\int_{\sigma}^{\sigma_{\max }} d \sigma_{c} \int_{\sigma_{\min }}^{\sigma} \tilde{\rho}\left(\sigma_{c}, \sigma_{o}\right) d \sigma_{o} .
$$


Here, $\varepsilon^{\uparrow}(\sigma)$ and $\varepsilon^{\downarrow}(\sigma)$ respectively represent the strain value at a compression $\sigma$ when loading or unloading the sample within the particular hysteretic loop. From the above equation it readily follows that

$$
\tilde{\rho}\left(\sigma_{\max }, \sigma\right)=\frac{\partial^{2} \Delta \varepsilon}{\partial \sigma_{\max } \partial \sigma} .
$$

The conventional practice of inferring the PM density thus implies the second derivative of the loop thickness information in the experimental stress-strain curves. Since experimental stress-strain curves are usually subject to substantial noise, this differential operation is badly conditioned. We can avoid the differential density reconstruction by working with the integral formulation.

In the integral approach, it suffices to measure the thicknesses $\Delta \varepsilon$ from the available stress-strain data for all $\sigma$-levels at the grid points in the PM space. For an $N \times N$ grid with $N_{L}=N(N+1) / 2$ possible triangles (i.e. loops in the stress-strain relation) and the corresponding $N$-level stress protocol, the maximum number of possible "nonzero" thickness measurements is $N_{M}=N\left(N^{2}-1\right) / 6$. This means that each $N$-level protocol gives us the PM density integrals over $N_{M}$ rectangles, overlapping as well as nonoverlapping. Once these values are known, it is possible to infer the integrals over the individual $1 \times 1$ squares in the PM space (which we call blocks or bins). The number of such bins is $N_{B}=N(N-1) / 2$. Remark that the number of unknown bin integrals $N_{B}$ is always smaller then the number $N_{M}$ of known data points (i.e. loop thickness measurements, or rectangles (bins combinations)), except in the trivial case for $N=2$, when both numbers equal 1. As a consequence, we have an over-determined system of linear equations that can be solved by minimizing the corresponding objective function: 


$$
F=\sum_{i=1}^{N_{M}}\left(\Delta \varepsilon_{i}-\gamma \sum_{k, l \in i} I_{k, l}\right)^{2}
$$

where $\Delta \varepsilon_{i}$ is the result of the $i$-th thickness measurement and $\sum_{k, l \in i} I_{k, l}$ represents the summation of all bin integrals (i.e., PM density integrals over the $(k, l)^{\text {th }}$ bin) belonging to the corresponding $i$-th rectangle. The residual value of the objective function can be used as a criterion for deciding whether we are dealing with a good PM system or a system that significantly deviates from the ideal one. (Note that an ideal PM system is defined by Eqs. $(1-2)$ or $(1,4)$, and its properties can by found in the classical article by Mayergoyz [3] or in the fundamental book by Krasnosel'skii and Pokrovskii [2].

The problem of calculating the individual bins values from their combinations basically requires the subtraction of two closely valued positive numbers, each known with some precision. In that case, the relative error of the result is much higher than the relative errors of the individual components. In other words, the calculation of the individual bins values is a coarse-grid equivalent of the continuous differentiation given by Eq. (7). However, in our current approach, we don't really need the bin values themselves, but only a specific combination of them corresponding to the grid points where the IPM density is calculated. To find the IPM density in a given grid point $\left(\sigma_{c}, \sigma_{o}\right)$ we must collect all bins located at the upper-left hand side from that point (see Figure 4). By summing bin integrals, we avoid the above mentioned instability problem since the sum of positive numbers always has a small relative error. In other words, we calculate a combination of items from another combination of items, that's why the requirements to error on each item are not too high. 
Note that, despite the fact that we combine bins in the IPM reconstruction algorithm, it is advisable to assure the stability of the process of deriving the bins themselves. A typical regularization technique [7] consists in considering a stabilizing addition to the objective function (Eq. 8) of the following kind

$$
F^{\prime}=F+\alpha \sum_{k, l}\left(I_{k, l}-I_{k+1, l}\right)^{2}+\alpha \sum_{k, l}\left(I_{k, l}-I_{k, l+1}\right)^{2}
$$

which penalizes large differences between neighboring bins and smoothens the coarsegrained PM density. The same consideration was made by Guyer et al. [6], however, with one major substantial difference. In this article the proposed smoothening is of vital importance for finding the differential density, while here it represents just a small correction that is not critical at all. The stabilizing parameter $\alpha$ must be predetermined depending on the noise value (as will be discussed later).

As presented in Figure 4, the IPM density $H\left(\sigma_{c}, \sigma_{o}\right)$ consists of elementary square bins and small triangular bins which are located at the diagonal $\sigma_{o}=\sigma_{c}$. To estimate the integrals in the triangular bins, we assume that the PM density in each triangular bin has a constant value ( $N$ in total). This is, of course, not exactly true and may lead to errors which will be discussed later. From Eq. (6), it follows that the area inside any closed stress-strain loop, extending from $\sigma_{\min }$ to $\sigma_{\max }$ under the assumption of a constant PM density $\tilde{\rho}_{0}$, is

$$
A=\int_{\sigma_{\min }}^{\sigma_{\max }} \Delta \varepsilon d \sigma=\tilde{\rho}_{0}\left(\sigma_{\max }-\sigma_{\min }\right)^{3} / 6 .
$$


Thus, by measuring the areas $A$ for all the smallest stress-strain loops, one easily obtains the values of the triangular bin integrals:

$$
\tilde{\rho}_{0} \frac{\left(\sigma_{\max }-\sigma_{\min }\right)^{2}}{2}=3 \frac{A}{\left(\sigma_{\max }-\sigma_{\min }\right)}
$$

Here $\tilde{\rho}_{0}, \sigma_{\max }$ and $\sigma_{\min }$ are, of course, different for different triangular bins.

With this, we have all information for obtaining the IPM density $H\left(\sigma_{c}, \sigma_{o}\right)$ at the discrete grid points. Finally, we interpolate the set of IPM points with a smooth function to get the IPM density distribution everywhere in the $\left(\sigma_{c}, \sigma_{o}\right)$ space. Inversely, this distribution can then again be used to predict the hysteretic contribution to the total strain following any arbitrary stress protocol.

We now return to the nonhysteretic contribution to the total strain. The component $\varepsilon_{N H}(\sigma)$ in Eq. (1) can only be reconstructed once the IPM density is found. To do this, we consider the loading curve $\varepsilon^{\uparrow}(\sigma)$ for the largest loop of the protocol and we note that

$$
\varepsilon^{\uparrow}(\sigma)=\varepsilon_{N H}(\sigma)+H\left(\sigma, \sigma_{\min }\right)
$$

Alternatively, we can also consider the unloading curve for which

$$
\varepsilon^{\downarrow}(\sigma)=\varepsilon_{N H}(\sigma)+H\left(\sigma_{\max }, \sigma_{\min }\right)-H\left(\sigma_{\max }, \sigma\right)
$$

Using both the measured loading and unloading branches and with the known IPM density values at hand, we can retrieve $\varepsilon_{N H}(\sigma)$ twice. Certainly, for an ideal PM system 
these two values must coincide. For realistic cases, they deviate with a discrepancy characterizing the validity of the discussed PM model.

The performed numerical analysis enabled us to choose the number $N$ from the following compromise consideration: on one hand, to coarse grid hides local features of the density, but, on the other hand, too fine grid makes it difficult to measure thickness of the smallest internal loops. $N=8$ was established as a optimum in our situation.

In conclusion, the algorithm for the IPM density computation and nonlinear elasticity calculation can be summarized in the following seven steps:

1. Choose $N$ and execute the corresponding $N$-level stress protocol depicted in Figure 2.

2. Measure the thickness of all loops at the grid points.

3. Minimize the objective function (Eq. 9) to get all square bins.

4. Measure the areas of the smallest loops to get all triangular bins.

5. Collect all bins related to the IPM density at the grid points.

6. Interpolate the discrete set of IPM points to obtain the IPM density distribution everywhere in the $\left(\sigma_{c}, \sigma_{o}\right)$ space.

7. Determine the non-hysteretic contribution.

In the next Section, we will illustrate the use of this method for simulated data, and discuss how to choose the optimal values for certain parameters used in the algorithm.

\section{Testing the algorithm with synthetic data}

In order to test the proposed method and illustrate the IPM density reconstruction, including errors, we performed the following actions: 
1. We assume a particular analytical model form for the PM (or IPM) density.

2. We compute the response (stress-strain loops) for an 8-level stress protocol by means of Eq. (4).

3. We accept a particular noise model and contaminate the stress-strain response with noise of given amplitudes.

4. We approximate the noisy stress-strain data by piecewise polynomials of a given degree.

5. We reconstruct the IPM density using the algorithm discussed in section 3, and calculate the global reconstruction error by comparing the result with the assumed analytical IPM density.

6. We vary certain parameters of the algorithm in order to minimize this error.

7. We repeat actions 3-6 for different noise amplitudes.

The above detailed plan makes our method and its results fully reproducible by the reader.

The particular model form we assumed for the verification of the reconstruction model is a two-dimensional Gaussian distribution of the PM density:

$$
\tilde{\rho}\left(\sigma_{c}, \sigma_{o}\right)=\tilde{\rho}_{m} \exp \left(-\frac{\left(\sigma_{c}-\sigma_{o}\right)^{2}}{4 d_{\perp}^{2}}\right) \exp \left(-\frac{\left(\sigma_{c}+\sigma_{o}\right)^{2}}{4 d_{\|}^{2}}\right)
$$

where the parameters $d_{\perp}$ and $d_{\|}$account for the widths of the distributions perpendicular and parallel to the diagonal $\sigma_{o}=\sigma_{c}$, and $\tilde{\rho}_{m}$ is a normalization constant whose value is not critical for the discussion here. For this particular formulation, we numerically computed the IPM density $H\left(\sigma_{c}, \sigma_{o}\right)$ given by Eq. 3, and obtained, using Eq. 4, the hysteretic component of the stress-strain curve for an 8-level stress protocol 
(see Figure 2). For the non-hysteretic component we choose a simple first order nonlinear $\sigma-\varepsilon_{N H}$ dependence

$$
\sigma=E_{o} \varepsilon_{N H}\left(1+\beta \varepsilon_{N H}\right)
$$

where $E_{o}$ denotes the static Young's modulus and $\beta$ reflects a first order elastic nonlinearity parameter. In the simulation we have set $E_{o}=5000 \mathrm{MPa}$ and $\beta=380$. Inversely, this corresponds to the nonlinear relation $\varepsilon_{N H}=1.316 \cdot 10^{-3} \cdot(\sqrt{1+0.304 \sigma}-1)$, with $\sigma$ expressed in MPa. This expression is based on a fit of real experimental data, but is not critical to the present study since the IPM reconstruction algorithm works with loop thicknesses, thereby eliminating the reversible contributions.

In order to simulate real experimental conditions, we introduced noise contamination on the numerical stress-strain data. In our simulation, we used a model of purely additive noise:

$$
\varepsilon^{\prime}=\varepsilon+\eta \xi
$$

where $\varepsilon$ is the numerically calculated value of the strain response, $\varepsilon^{\prime}$ is the strain value contaminated with noise, $\eta$ is the noise amplitude (an instrumental constant), and $\xi$ is a random number with Gaussian distribution of zero mean and unit variance. The choice of the noise model resulted from a comparison of experimental and simulated stressstrain data. Using the additive noise model, the behavior of the polynomial approximation error versus loop number for real data and for simulated data subjected to Eq. (16) were found to be quite similar (we omit the graphs for brevity). 
In an additive noise model, smaller loops are more drastically affected by the noise than larger loops. Figure 5 illustrates a typical case for $\eta=310^{-5}$. The loop corresponding to a small excursion with stress amplitude $\sigma_{\max }-\sigma_{\min }$ equal to $12.5 \%$ of the total stress excursion (50 MPa) almost completely loses its shape, while a big loop with $\sigma_{\max }-\sigma_{\min }=50 \mathrm{MPa}$ is still clearly discernible and reproducible. As a result, it is reasonable to use a different (smaller) degree of polynomials to approximate the smallest loops.

In order to reconstruct the IPM density, we first approximated the noisy stressstrain data by piecewise polynomial functions of degree $N_{p 1}$ for the smallest loops and $N_{p 2}$ for the other loops. Next, we determined the thicknesses of all loops except for the smallest ones. For the smallest ones, we measured their areas. If due to the presence of noise, some lengths or areas are found to be negative, we assign a zero value.

The obtained values are then transformed into the IPM density using the aforementioned procedure. Figure 6 illustrates the resulting reconstructed IPM density, $H_{\text {rec }}\left(\sigma_{c}, \sigma_{o}\right)$, smoothed by a $2 \mathrm{D}$ spline interpolation, corresponding to the case with a noise amplitude $\eta=3 \cdot 10^{-5}$, and the corresponding local reconstruction error $\left|H\left(\sigma_{c}, \sigma_{o}\right)-H_{\text {rec }}\left(\sigma_{c}, \sigma_{o}\right)\right|$. In some regions close to the diagonal $\sigma_{o}=\sigma_{c}$, the noisy data may lead to very small negative IPM densities in the spline approximated distribution. The underlying reason for the negative values, which are obviously not physical, is purely of mathematical nature. Even though we assume an anti-symmetric extension of the IPM density into the half-plane $\sigma_{o}>\sigma_{c}$, i.e. $H_{r e c}\left(\sigma_{c}, \sigma_{o}\right)=-H_{r e c}\left(\sigma_{o}, \sigma_{c}\right)$, small negative values may appear as approximation errors, since the anti-symmetry axis $\sigma_{o}=\sigma_{c}$ is angled $45^{\circ}$ with respect to the IPM space grid lines. In any case, we have found that these approximation errors increase the reconstruction error only slightly. An alternative assumption which sets $H_{r e c}\left(\sigma_{c}, \sigma_{o}\right)=0$ for $\sigma_{o}>\sigma_{c}$ does not greatly affect the situation. 
The reconstruction algorithm uses several parameters: the degrees of the polynomials $N_{p 1}$ and $N_{p 2}$ for the approximation of the stress-strain loops (the smallest and the others, respectively), the regularization parameter $\alpha$ (see Eq. (9)) for the bins, and the spline order $N_{s}$ for the final 2D IPM approximation of the gridded data. Their role has been investigated in terms of the optimization of the reconstruction. The main criterion in the parameter's optimization is the final global IPM reconstruction error for the inverted and spline approximated distribution in comparison with the exact IPM distribution, i.e. $R M S\left[H\left(\sigma_{c}, \sigma_{o}\right)-H_{r e c}\left(\sigma_{c}, \sigma_{o}\right)\right] / R M S\left[H\left(\sigma_{c}, \sigma_{o}\right)\right]$. The least squares reconstruction error was computed on a $700 \times 700$ mesh in the $\left(\sigma_{c}, \sigma_{o}\right)$-space, restricted to the half space $\sigma_{c}<\sigma_{o}$. (As the final form of the reconstructed IPM density is given by a spline, it is possible to calculate the analytical function at any $\left(\sigma_{c}, \sigma_{o}\right)$-point.) In addition, we also calculated the least squares reconstruction errors for the individual bin integrals and for the gridded IPM data (again, in comparison with the exact values).

The values shown in Figure 7 represent the three above mentioned errors for different noise amplitudes and for optimized algorithm parameters. One clearly observes that the global reconstruction error on the bin values is much higher compared to the gridded and spline interpolated IPM reconstruction errors. We expected this beforehand, since the reconstruction of the bin values is a coarse grid equivalent solution of the ill-posed problem of the PM density reconstruction. This graph confirms that the IPM inversion is well-posed.

With respect to the reversible elasticity parameters, as listed in Eq.(15), we obtained an error on the static Young's modulus $E_{o}$ of $0.9 \%$ for the largest noise level, and an error of $4 \%$ for the first order nonlinear coefficient $\beta$.

Numerous numerical experiments lead us to the following general conclusions about the optimal parameter values. 
- The choice of the spline order $N_{s}$ does not influence the bin and gridded IPM errors. For $N_{s}=3$ the additional error introduced by the splines is minimal, at all noise amplitudes.

- For polynomial degrees $N_{p 1}$ and $N_{p 2}$ we recommend the values $N_{p 1}=3$ and $N_{p 2}=6$, again for all noise amplitudes.

- The optimum regularization parameter $\alpha$ depends on the noise amplitude $\eta$ (in accordance with the general theory by Tikhonov and Arsenin [7]). Nevertheless, the global error is not crucially sensitive to $\alpha$, since $\alpha$ serves as an internal regularization only, which mainly affects the bin values.

Table 1 lists the recommended values of $\alpha$ for different $\eta$. The use of an $\alpha$ value which deviates by 50 or even $100 \%$ from the recommended value is often not critical. For instance, for $\eta=4 \cdot 10^{-5}$ the value $\alpha=1.5$ (optimal) leads to a global reconstruction error of $4.0 \%$ while at $\alpha=0.5$ the error equals $4.2 \%$. However, if one takes $\alpha=10$ then the global reconstruction error grows to $7.1 \%$. So, we may conclude that only orders of $\alpha$-values are essential.

A proper choice of the degree $N_{p 1}$ is more important: for example, for $N_{p 1}=4$ instead of 3 (optimal), the global error easily increases by $0.3-0.8 \%$ for noisy data $\left(\eta>1 \cdot 10^{-5}\right)$.

Strictly speaking, the magnitude of the reconstruction error may vary for different values of the PM density widths $d_{\perp}$ and $d_{\|}$(see Eq. 14), but our numerical tests have shown that the general recommendations hold. Here we used $d_{\perp}=30 \mathrm{MPa}$ and $d_{\|}=50 \mathrm{MPa}$, which correspond to the differential PM density shown in the 2D grayscale plot of Figures 2 to 4 . Smaller value of the widths will generally increase the error since 
the assumption of a constant PM density inside each of the triangular bins then is violated more easily.

\section{IPM density reconstruction from experimental data}

We now apply the reconstruction method to real experimental data resulting from uniaxial compression tests on natural building stones. We have analyzed the following rock materials: Serena sandstone, Sander sandstone, Vicenza limestone, Lecce limestone and Portland limestone. Some physical properties of these rocks are listed in Table 2. Among other natural stones, these materials were extensively tested in laboratory conditions within the EU-FP5 grant DIAS [15] for comparison with the results of a combination of three prototype devices (micro-drilling, micro-indentation and Rayleigh wave acoustics) developed for in-situ characterization of their mechanical and acoustical parameters in historical buildings.

After having determined the uniaxial compression strength (UCS) for each rock type, we instrumented cylindrical samples (with a length of about $50 \mathrm{~mm}$, and a diameter of $25 \mathrm{~mm}$ ) with 2 dynamic strain gauge extensometers (Instron 2620-603 with gauge length $10 \mathrm{~mm}($ Ext1) and $25 \mathrm{~mm}($ Ext2) and full-scale range $\pm 1 \mathrm{~mm})$ and placed them between the grips of a MTS 810 TestStar load frame (Figure 8). This system is capable of loading up to $100 \mathrm{kN}$. The MTS software allows to translate custom designed command signals, such as our 8-level stress protocol. The experiments are load-driven and can be controlled very accurately (error between command and actual loading is less than $0.025 \mathrm{kN}$ ). The tests on the different samples are performed with the same speed $(0.125 \mathrm{kN} / \mathrm{s})$. For each rock type, we define the loading maximum $\sigma_{60} \%$ for the 8 - 
protocol as $60 \%$ of the material's strength at failure. During the experiment, both extensometers and the actual loading force are registered every $0.2 \mathrm{~s}$.

Figure 9 illustrates the complete stress protocol which is loaded on the experimental device. It contains three parts which are executed without delay in between. In the first part, we initialize the complete system by performing ten cycles of $60 \%$ of the maximum stress loading and unloading. The initialization assures that the stress-strain behavior becomes repeatable. In the second part, the 8-level stress protocol is executed. Finally, we continue with additional loading-unloading commands which are used to test the IPM reconstruction. The first "conditioning" loops and the final "additional" loops are not used in the reconstruction algorithm. Only the second part between 5000 and 13000 seconds is used for the inversion.

Figure 10, similarly to Figure 5 for the synthetic data, illustrates two loops of the stress-strain curve for Serena sandstone $\left(\sigma_{60 \%}=58 \mathrm{MPa}\right)$. The large loop $\left(0 \rightarrow \sigma_{60 \%} \rightarrow 0\right)$ switches all the hysteretic elements contained in the covered pressure range. This loop evidently shows the largest difference between loading and unloading curves. The inset represents one of the smallest loops obtained at the highest ambient pressure $(58 \mathrm{MPa} \rightarrow 51 \mathrm{MPa} \rightarrow 58 \mathrm{MPa})$. At these pressures almost all hysteretic movement (in compression or shear) of crack surfaces inside the rock is disabled [16], and the loop becomes considerably less hysteretic than the others. Correspondingly, the two loops in Figure 10 are the most and the least hysteretic ones within the protocol.

The largest loop $(0 \rightarrow 58 \mathrm{MPa} \rightarrow 0)$ was used to retrieve the non-hysteretic contribution $\varepsilon_{N H}(\sigma)$ according to Eqs. 12-13. The results are shown in Figure 11. As mentioned, the algorithm enables to reconstruct the non-hysteretic dependence twice, based on either the up-going or the down-going branches of the largest loop. Good 
coincidence between the inverted data points (large and small crosses) confirms the validity of the PM model for this material.

In Figure 12 we have plotted two IPM portraits, one for a sandstone (Sander) and one for a bioclastic limestone (Lecce). The petrographic parameters (type and size of grains, pores and binding material) of these two rocks are considerably different, as well as their physical properties. In particular, as can be seen from Table 2, the porosity, grain size, and UCS values all differ about an order of magnitude. The reconstructed hysteretic portraits of both rocks deduced from their corresponding experimental stressstrain curves are found to be considerably different too: the IPM densities have distinctive shapes. We observe that the contour lines for the IPM density of Sander sandstone are more inclined with respect to the diagonal than for Lecce limestone. This means that Serena sandstone has relatively more hysteretic features with low opening pressures.

Figure 13 summarizes all reconstructed IPM portraits for the rocks considered in the present study. We clearly see that the two sandstones display a similar structure of the IPM distribution with rather different characteristics compared to the three limestones. Some characteristic parameters of the IPM-distributions are listed in Table 3. The maximum IPM density $H_{\max }$ corresponds to the hysteretic contribution to the strain $\varepsilon_{\mathrm{H}, \text { max }}$ at $60 \%$ of the ultimate compression strength $\left(\sigma_{60 \%}\right)$. We notice that these values are comparable for rocks of the same types: around $8 \cdot 10^{-4}$ for the class of the 'harder' sandstones, and from 0.6 to $2 \cdot 10^{-4}$ for the class of the 'softer' limestones. Interestingly enough, the normalized parameter $H_{\max } / \sigma_{60 \%}{ }^{2}$ which characterizes the total "amount" of hysteresis per unit of pressure-pressure space does not show the same classification tendency. This means that apparently similar portraits for rocks of the same class, despite the visual similarity, can have significantly varying quantitative 
parameters. We have also found that, if properly prepared, two samples of the same rock produce the same IPM densities within their error intervals.

The 2D information in the IPM density distribution makes it hard to directly compare quantities between the rocks. On the other hand, simple numerical parameters such as the ones listed in Table 3 carry severely limited information on the full reconstructed portraits. For a quick and meaningful understanding of the differences between the rocks, we compared particular 1D profiles expressed in normalized variables in Figure 14. We therefore considered the two sections $H\left(\sigma_{c}, \sigma_{o}=0\right)$ and $H\left(\sigma_{c}=\sigma_{60 \%}, \sigma_{o}\right)$. Note that, despite the reduction of the number of arguments, both characteristics are not "local" but rather integral quantities, since they cover all hysteretic elements contained in particular triangles with only one varying side. In fact the two profiles simply correspond to the hysteretic strain contributions on the loading and unloading branches of the largest loop. In order to compare the comportment for different rocks, we first normalized these dependencies, $H\left(\sigma, \sigma_{o}=0\right) / H_{\max }$ and $H\left(\sigma_{c}=\sigma_{60 \%}, \sigma\right) / H_{\max }$, and plotted them as function of $\sigma / \sigma_{60 \%}$ in Figure 14 for all the rocks under test. Certainly, the values $\sigma_{60 \%}$ and $H_{\max }$ used in the definition of the section and in the normalization bring in a certain influence of the arbitrary parameter of $60 \%$ UCS. Nevertheless, in a similar manner as in the IPM portraits, rocks of the same types tend to group around similar profiles. In conclusion, we can say that the normalized 1D curves, although suffering from the point of generality, offer a simple means for the comparison of the hysteretic properties.

In an analogous manner, we analyzed the non-hysteretic contribution $\varepsilon_{N H}(\sigma)$ to the total strain for the different rocks using Eq. (12) and/or (13). When normalized to its maximum at $\sigma_{60 \%}$ and expressed in terms of the normalized stress value $\sigma_{d} / \sigma_{60 \%}$, we can observe the same clustering of the two groups, as illustrated in Figure 15. In addition, 
we used the first order nonlinear expression of the non-hysteretic stress-strain relation (Eq. 15) to quantify the values of the quasi-static Young's Modulus $E_{0}$ and the first order nonlinearity coefficient $\beta$. The values are listed in Table 4 and, for the Young's modulus, they are compared to the values obtained from dynamic velocity measurements which were performed on similar cylindrical samples using resonance spectroscopy. The observation is that the static modulus is generally smaller than the dynamic modulus and that the limestones have a smaller 'classical' first order nonlinearity coefficient compared to the sandstones.

As a final point in the experimental analysis, we also investigated the predictive power of the considered methodology. As mentioned before, the final part of the executed protocol (Figure 9, gray line for times later than 13000 seconds) was not used for the construction of the system's IPM portrait. It was added to the protocol in order to serve as a test for the stress-strain prediction. Figure 16 illustrates the actual measurement of the stress-strain curve together with the predicted behavior that was calculated using Eq. (1), Eq. (4) and the reconstructed IPM density for Sander sandstone (Figure 13). The agreement is most satisfactory. The experimental and predicted shapes only weakly deviate from each other: the relative error of the deviation $\left(\operatorname{RMS}\left(\varepsilon_{p, i}-\varepsilon_{\text {exp }, i}\right) / \operatorname{RMS}\left(\varepsilon_{\text {exp }, i}\right)\right)$ is $2 \%$. The major difference is a constant shift, which can be attributed to the fact that successive cycles are never perfectly repeatable. Even after a long initialization process, a small shift of the successive loops remains present in the experiment. 


\section{Conclusions}

We introduced the IPM density as a means for characterizing the hysteretic mechanical properties of solid materials and showed that it is a robust concept, even in the presence of noise. The reconstruction of the IPM density is based on a particular force protocol and involves simple mathematical data treatment. The methodology has been illustrated for synthetic data and applied for the characterization of several classes of natural building stones. The reconstruction of the IPM density and the analysis of the nonhysteretic stress-strain contribution revealed that sandstones are more hysteretic and more nonlinear than limestones in the compressive stress range of 0 to $60 \%$ of the limiting strength. The physical reason for this difference is to be found in the different composition and binding properties of the two classes.

Even though a limiting stress value of $60 \%$ of the UCS was used, the protocol and methodology can be executed for any arbitrary stress range. The resulting IPM density then enables us to predict any stress-strain relation within this range with great accuracy.

In the future, we intend to characterize the influence of damage mechanisms on the IPM density of various materials by investigating the alterations in the distribution characteristics as the result of progressive thermal and/or mechanical fatigue loading. This may for instance lead to a very interesting application in the quantitative evaluation of the effect of fire damage on concrete walls, housing, tunnels, etc.

In addition we plan to perform an intense comparative study between the static nonlinear and hysteretic elasticity, and the dynamic nonlinear properties of the same material. This will inform us about the link, or the absence of a link, between the two strain ranges. 


\section{Acknowledgments}

The authors gratefully acknowledge the support of the Flemish Fund for Scientific Research. (G.0206.02, G.0257.02, and G.0554.06), the provisions of the European Science Foundation Program NATEMIS and the European FP5 and FP6 Grants DIAS (EVK4-CT-2002-00080) and AERONEWS (AST3-CT-2003-502927). 


\section{References}

1. F. Preisach, Über die magnetische Nachwirkung. Z. Phys. 94, 277 (1935).

2. M.A. Krasnosel'skii and A.V. Pokrovskii, Systems with Hysteresis. Nauka, Moscow (1983).

3. I.D. Mayergoyz, Hysteresis models from the mathematical and control theory points of view. J. Appl. Phys. 57(1), 3803-3805 (1985).

4. J. Ortin, Preisach modeling of hysteresis for a pseudoelastic $\mathrm{Cu}-\mathrm{Zn}-\mathrm{Al}$ single crystal. J. Appl. Phys. 71(3), 1454 (1992).

5. K.R. McCall and R.A. Guyer, Equation of state and wave propagation in hysteretic nonlinear elastic materials. J. Geophys. Res. 99, 23887-23897 (1994).

6. R. Guyer, K. McCall, G. Boitnott, L. Hilbert Jr., T. Plona, Quantitative implementation of Preisach-Mayergoyz space to find static and dynamic elastic moduli in rock. J. Geophys. Res. 102 (B3), $5281-5293$ (1997).

7. A.N. Tikhonov and V.A. Arsenin, Solutions of Ill-posed Problems. Winston \& Sons, Washington (1977).

8. L. D. Landau and E. M. Lifshitz, Theory of Elasticity, 2nd edition. Pergamon Press, Oxford (1970).

9. C. Pecorari, Nonlinear interaction of plane ultrasonic waves with an interface between rough surfaces in contact. J. Acoust. Soc. Am. 113, 3065-3072 (2003).

10. C. Pecorari, Adhesion and nonlinear scattering by rough surfaces in contact: Beyond the phenomenology of the Preisach-Mayergoyz framework, J. Acoust. Soc. Am. 116(4), 1938-1947 (2004).

11. V. Aleshin and K. Van Den Abeele, Micro-potential model for stress-strain hysteresis of micro-cracked materials. J. Mech. and Phys. Solids 53(4), 795-824 (2005). 
12. V. Aleshin and K. Van Den Abeele, Microcontact based theory for acoustics in microdamaged materials. J. Mech. and Phys. Solids 55, 366-390 (2007).

13. V. Aleshin and K. Van Den Abeele, Friction in unconforming grain contacts as a mechanism for tensorial stress-strain hysteresis. J. Mech. and Phys. Solids 55, 765 787 (2007).

14. K. Van Den Abeele, P.Y. Le Bas, B. Van Damme, T. Katkowski and C. Mattei, Nonlinearity and crack density in thermally loaded CFRP, in preparation.

15. Integrated tool for in situ characterization of effectiveness and durability of conservation techniques in historical structures (DIAS), EU-FP5 Project (RTD EVK4-2001-00141), http://minelab.mred.tuc.gr/dias/

16. G. Mavko, Frictional attenuation: An inherent amplitude dependence. J. Geophys. Res. 84, 4769-4777 (1979). 


\section{Figure captions}

Figure 1. Schematic representation of areas of closed (dark gray, $\Omega_{c}$ ) and open (light gray, $\left.\Omega_{o}\right)$ hysteretic units in a PM space $\left(\sigma_{c}, \sigma_{o}\right)$, separated by a staircase line $(1,2,3$, $\ldots n)$.

Figure 2. Specific $N$-level stress protocol (with $N=8$ ) used in the reconstruction algorithm (left figure). Each individual loading-unloading cycle uniquely corresponds to one of the possible triangles composed by any two grid lines shown at the right hand side.

Figure 3. Illustration of the strain thickness measure $\Delta \varepsilon$ at constant stress value $\sigma$ for a typical hysteretic loop in the stress-strain relationship (left figure) and the corresponding rectangle in the PM space (on the right). The density integral over this rectangle equals $\Delta \varepsilon$. The corners of the rectangle are defined by the highest and the lowest stress values of the loop as well as the stress $\sigma$ at which $\Delta \varepsilon$ is measured.

Figure 4. The IPM density at the marked grid point $\left(\sigma_{c}, \sigma_{o}\right)$ is the sum of the PM density integrals for all bins (square and triangular) in the hatched triangle.

Figure 5. The largest loop simulation of the 8 -level stress protocol $(0 \rightarrow 50 \mathrm{MPa} \rightarrow 0)$ and one of the smallest loops $(50 \mathrm{MPa} \rightarrow 43.75 \mathrm{MPa} \rightarrow 50 \mathrm{MPa})$ affected by the additive noise model (Eq. 16) with $\eta=3 \cdot 10^{-5}$. The solid lines represent polynomial approximation of $3^{\text {rd }}$ and $5^{\text {th }}$ degree for the small and the large loop, respectively. 
Figure 6 . The reconstructed IPM density $H_{r e c}\left(\sigma_{c}, \sigma_{o}\right)$, left, and the local reconstruction error, right, for synthetic data contaminated with additive noise $\left(\eta=3 \cdot 10^{-5}\right)$ based on the PM density given by Eq.(14) for $d_{\perp}=30 \mathrm{MPa}$ and $d_{\|}=50 \mathrm{MPa}$. The global reconstruction error is $3 \%$ for a regularization parameter $\alpha=0.5$, a $3^{\text {rd }}$ degree polynomial approximation of the smallest loops and a $6^{\text {th }}$ degree approximation for the others loops. Splines of the $3^{\text {rd }}$ order were used to retrieve the entire distribution function from the discrete IPM grid data.

Figure 7. Global reconstruction errors for different noise amplitudes $\eta$ using the optimized parameters of the algorithm. Black: global reconstruction error on the bin integrals; dark gray: global reconstruction error on the gridded IPM density; light gray: global reconstruction error on the spline approximated IPM density.

Figure 8. Experimental set-up for cyclic uniaxial compression tests on cylindrical rock samples.

Figure 9. Typical experimental stress command protocol, including initialization cycles (time $<5000 \mathrm{sec}), 8$-level stress protocol for reconstruction analysis $(5000<$ time $<$ $13000 \mathrm{sec}$ ), and additional cycling for validating the reconstruction (time $>1300 \mathrm{sec}$ ).

Figure 10. Experimental data corresponding to the largest loop of the 8-level stress protocol $(0 \rightarrow 58 \mathrm{MPa} \rightarrow 0)$ and of one of the smallest loops $(58 \mathrm{MPa} \rightarrow 51 \mathrm{MPa} \rightarrow 58 \mathrm{MPa})$ for a sample of Serena sandstone (each $20^{\text {th }}$ point is plotted). The solid lines represent 
polynomial approximation of $3^{\text {rd }}$ and $6^{\text {th }}$ degree for the small and the large loop, respectively.

Figure 11. Non-hysteretic contribution $\varepsilon_{N H}(\sigma)$ (discrete data points) and the largest hysteretic loop (solid lines) in the stress-strain behavior of Serena Sandstone. The small crosses correspond to the inversion of $\varepsilon_{N H}(\sigma)$ from the loading curve (Eq.12) and the large ones are obtained from the unloading curve (Eq. 13).

Figure 12. Reconstructed IPM density distributions for two different rocks (Serena sandstone and Lecce limestone).

Figure 13. Reconstructed IPM portraits for various rocks. In the right column: Serena and Sander sandstone. In the right column: Portland limestone, Vicenza limestone and Lecce limestone. The values $\sigma_{c}$ and $\sigma_{o}$ range from 0 to $\sigma_{60 \%}(60 \%$ of UCS $)$ for each rock. The maximum IPM density $H_{\max }$ corresponding to this interval is listed in Table 3 and defines the color scale of the plot.

Figure 14. Comparison of the normalized IPM densities at $\sigma_{o}=0$ and at $\sigma_{c}=\sigma_{60 \%}$ for different rocks. The thick black line represents Serena sandstone, the thick gray line is for Sander sandstone, the thin black line is for Vicenza limestone, the dotted line is for Lecce limestone, and the dashed line is for Portland limestone.

Figure 15. Comparison of the normalized non-hysteretic contribution for different rocks. Same line-style as in Figure 14. 
Figure 16. Prediction of additional loading-unloading cycles made for Sander sandstone. Black - actual experimental data; Gray - prediction based on the reconstructed IPM density. The relative RMS of the deviation between prediction and actual measurement is $2 \%$.

\section{Table captions}

Table 1. Recommended values of the regularization parameter $\alpha$ for different noise amplitudes.

Table 2. Material properties

Table 3. Maximum IPM density $H_{\max }$, maximum pressure $\sigma_{60 \%}$ of the protocol, and relative $H_{\max }$ related to the total pressure area $\sigma_{60 \%}{ }^{2}$ for different rocks.

Table 4. Linear and classical nonlinear moduli for different rocks. Comparison of the Quasi-Static (QS) Young's modulus (deduced from a first order polynomial expansion of the nonhysteretic contribution in the quasi-static stress strain relation) and the dynamic (Dyn) Young's modulus (deduced from velocity measurements at zero stress). 
Table 1: Recommended values of the regularization parameter $\alpha$ for different noise amplitudes.

\begin{tabular}{|l|l|l|l|l|l|l|l|l|l|}
\hline$\eta$ & 0 & $0.5 \cdot 10^{-5}$ & $1.0 \cdot 10^{-5}$ & $1.5 \cdot 10^{-5}$ & $2.0 \cdot 10^{-5}$ & $2.5 \cdot 10^{-5}$ & $3.0 \cdot 10^{-5}$ & $3.5 \cdot 10^{-5}$ & $4.0 \cdot 10^{-5}$ \\
\hline$\alpha$ & 0 & 0.02 & 0.06 & 0.1 & 0.15 & 0.3 & 0.5 & 1.0 & 1.5 \\
\hline
\end{tabular}


Table 2: Material properties.

\begin{tabular}{l|cccc} 
& $\begin{array}{c}\text { Density } \\
\left(\mathrm{kg} / \mathrm{m}^{3}\right)\end{array}$ & $\begin{array}{c}\text { Total } \\
\text { Porosity } \\
(\%)\end{array}$ & $\begin{array}{c}\text { Mean } \\
\text { Grain Size } \\
(\mu \mathrm{m})\end{array}$ & $\begin{array}{c}\text { UCS } \\
(\mathrm{MPa})\end{array}$ \\
\hline Serena sandstone & 2560 & 6.5 & $200-800$ & 95.8 \\
\hline Sander sandstone & 2200 & 19.7 & $150-200$ & 58.3 \\
\hline Portland limestone & 2030 & 20.1 & 300 & 31.4 \\
\hline Vicenza limestone & 1970 & 29.3 & $400-2000$ & 29.3 \\
\hline Lecce limestone & 1460 & 47.4 & $80-100$ & 11.0 \\
\hline
\end{tabular}


Table 3: Maximum IPM density $H_{\max }$, maximum pressure $\sigma_{60 \%}$ of the protocol, and relative $H_{\max }$ related to the total pressure area $\sigma_{60 \%}{ }^{2}$ for different rocks.

\begin{tabular}{l|ccc} 
& $H_{\max }(-)$ & $\sigma_{60 \%}, \mathrm{MPa}$ & $H_{\max } / \sigma_{60 \%}{ }^{2}, \mathrm{MPa}^{-2}$ \\
\hline Serena sandstone & $8.48 \cdot 10^{-4}$ & 58.0 & $2.56 \cdot 10^{-7}$ \\
\hline Sander sandstone & $7.55 \cdot 10^{-4}$ & 34.8 & $6.34 \cdot 10^{-7}$ \\
\hline Portland limestone & $1.86 \cdot 10^{-4}$ & 18.7 & $5.40 \cdot 10^{-7}$ \\
\hline Vicenza limestone & $5.92 \cdot 10^{-5}$ & 17.6 & $1.97 \cdot 10^{-7}$ \\
\hline Lecce limestone & $1.06 \cdot 10^{-4}$ & 6.50 & $2.65 \cdot 10^{-6}$ \\
\hline
\end{tabular}


Table 4. Linear and classical nonlinear moduli for different rocks. Comparison of the Quasi-Static $(Q S)$ Young's modulus (deduced from a first order polynomial expansion of the nonhysteretic contribution in the quasi-static stress strain relation) and the dynamic (Dyn) Young's modulus (deduced from velocity measurements at zero stress).

\begin{tabular}{l|ccc} 
& $\begin{array}{c}E_{0}-D y n \\
(G P a)\end{array}$ & $\begin{array}{c}E_{0}-Q S \\
(G P a)\end{array}$ & $\begin{array}{c}1^{\text {st }} \text { order } Q S- \\
\text { Nonlinearity } \beta(-)\end{array}$ \\
\hline Serena sandstone & 25.0 & 20.5 & 414 \\
\hline Sander sandstone & 15.7 & 14.0 & 1070 \\
\hline Portland limestone & 20.7 & 18.1 & 63 \\
\hline Vicenza limestone & 20.0 & 18.4 & 76 \\
\hline Lecce limestone & 8.6 & 9.1 & 125 \\
\hline
\end{tabular}




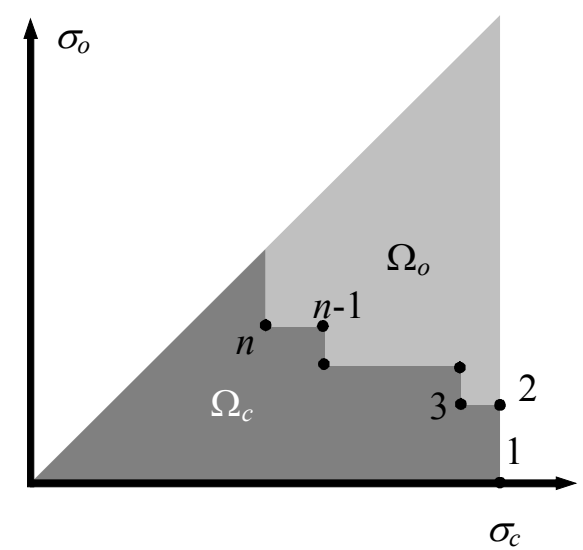

Figure 1. Schematic representation of areas of closed (dark gray, $\Omega_{c}$ ) and open (light gray, $\left.\Omega_{o}\right)$ hysteretic units in a PM space $\left(\sigma_{c}, \sigma_{o}\right)$, separated by a staircase line $(1,2,3$, ...n). 

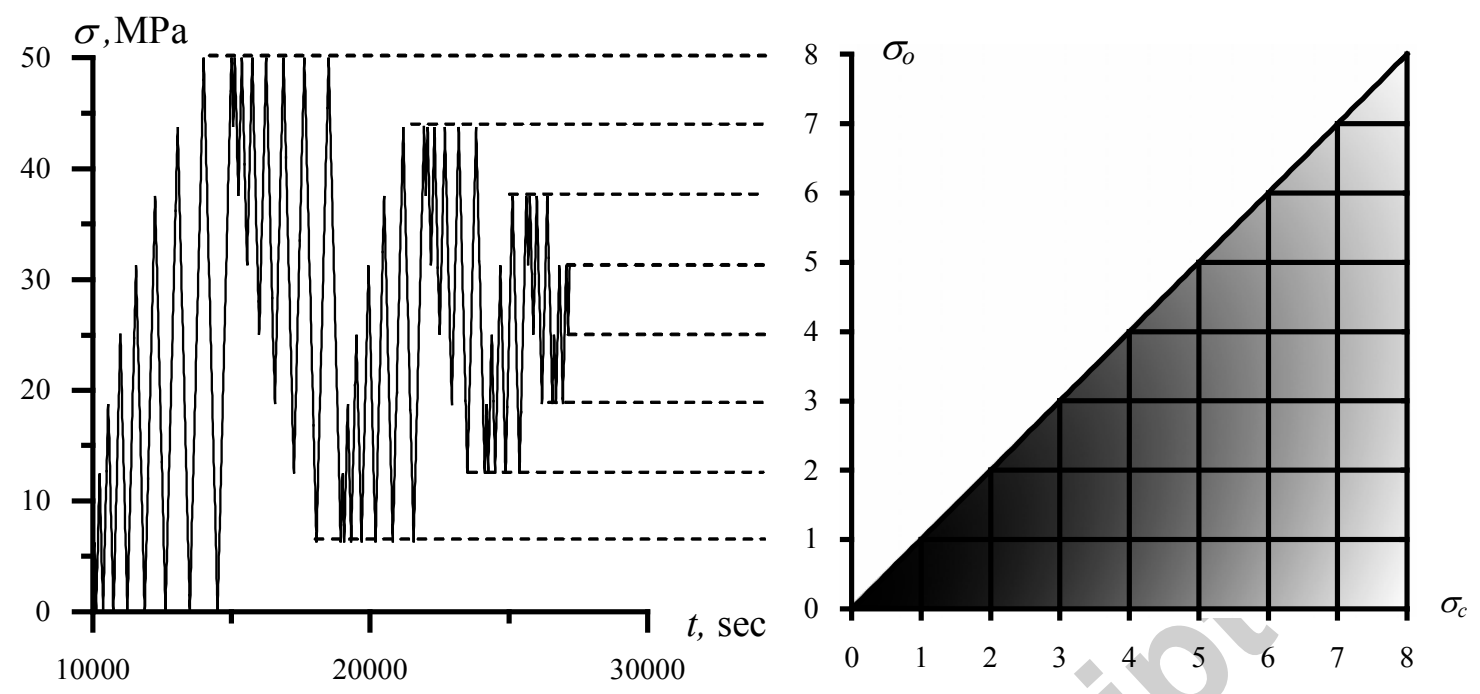

Figure 2. Specific $N$-level stress protocol (with $N=8$ ) used in the reconstruction algorithm (left figure). Each individual loading-unloading cycle uniquely corresponds to one of the possible triangles composed by any two grid lines shown at the right hand side. 


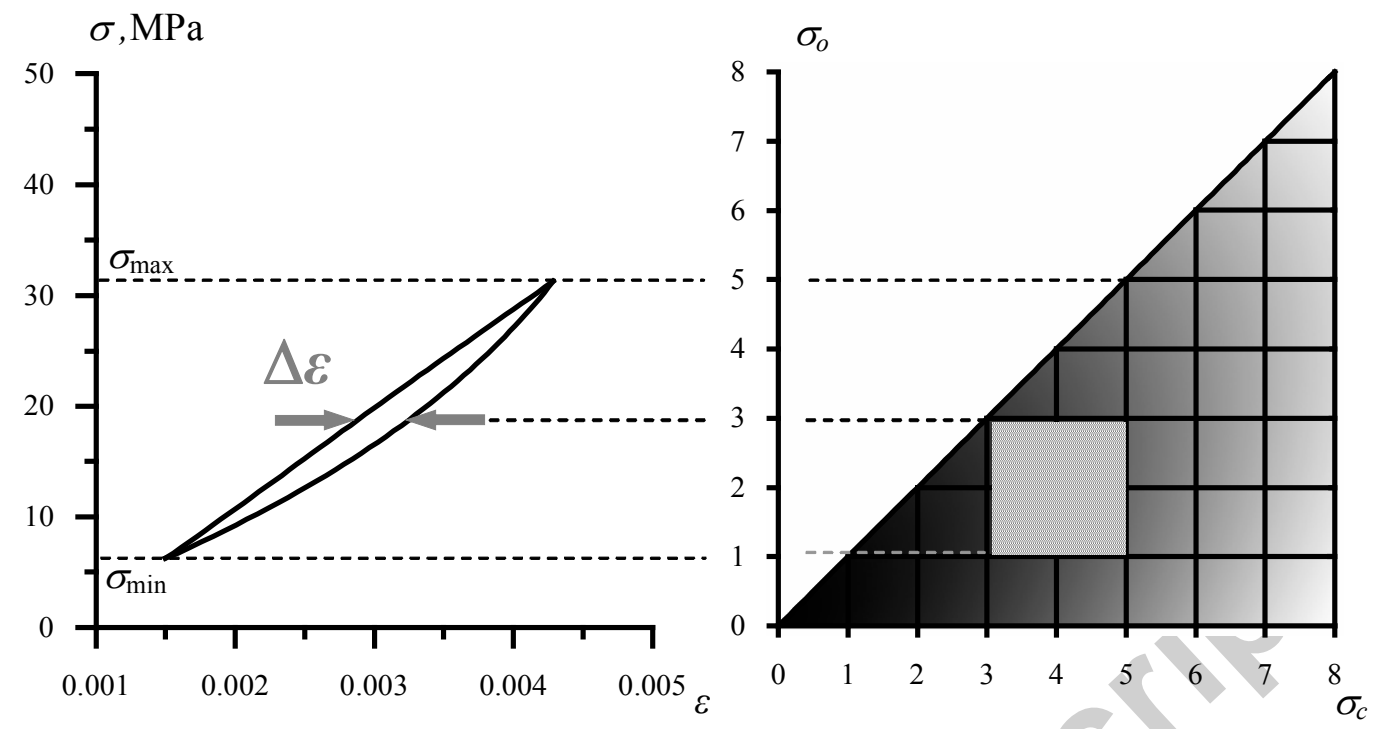

Figure 3. Illustration of the strain thickness measure $\Delta \varepsilon$ at constant stress value $\sigma$ for a typical hysteretic loop in the stress-strain relationship (left figure) and the corresponding rectangle in the PM space (on the right). The density integral over this rectangle equals $\Delta \varepsilon$. The corners of the rectangle are defined by the highest and the lowest stress values of the loop as well as the stress $\sigma$ at which $\Delta \varepsilon$ is measured. 


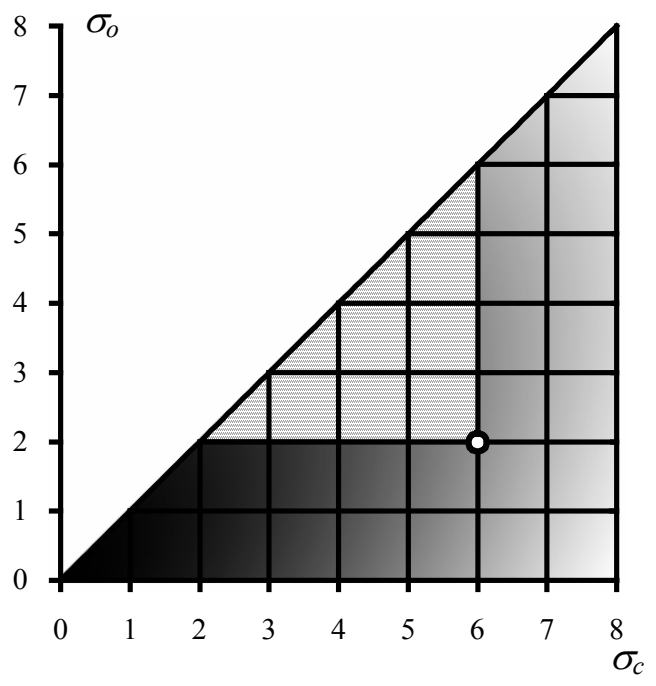

Figure 4. The IPM density at the marked grid point $\left(\sigma_{c}, \sigma_{o}\right)$ is the sum of the PM density integrals for all bins (square and triangular) in the hatched triangle. 


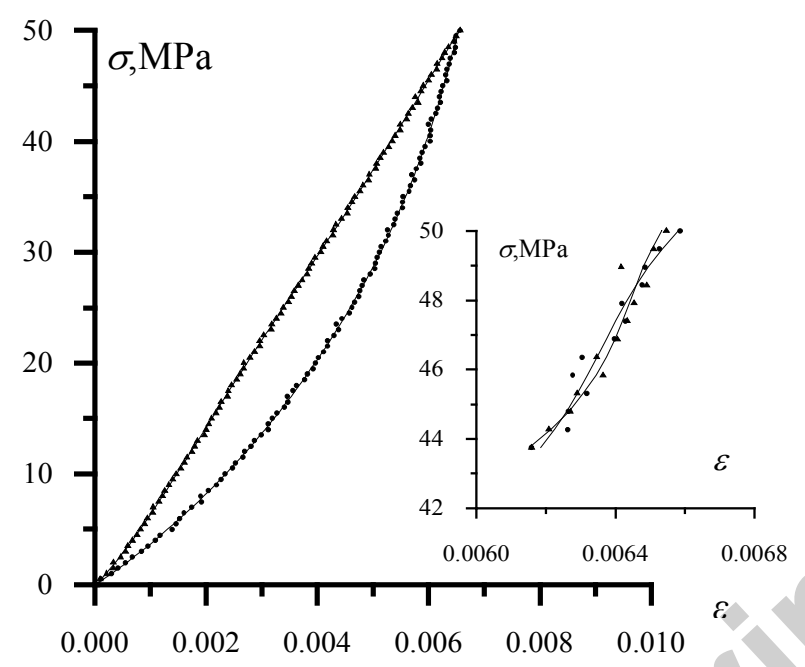

Figure 5. The largest loop simulation of the 8 -level stress protocol $(0 \rightarrow 50 \mathrm{MPa} \rightarrow 0)$ and one of the smallest loops $(50 \mathrm{MPa} \rightarrow 43.75 \mathrm{MPa} \rightarrow 50 \mathrm{MPa})$ affected by the additive noise model (Eq. 16) with $\eta=3 \cdot 10^{-5}$. The solid lines represent polynomial approximation of $3^{\text {rd }}$ and $5^{\text {th }}$ degree for the small and the large loop, respectively. 

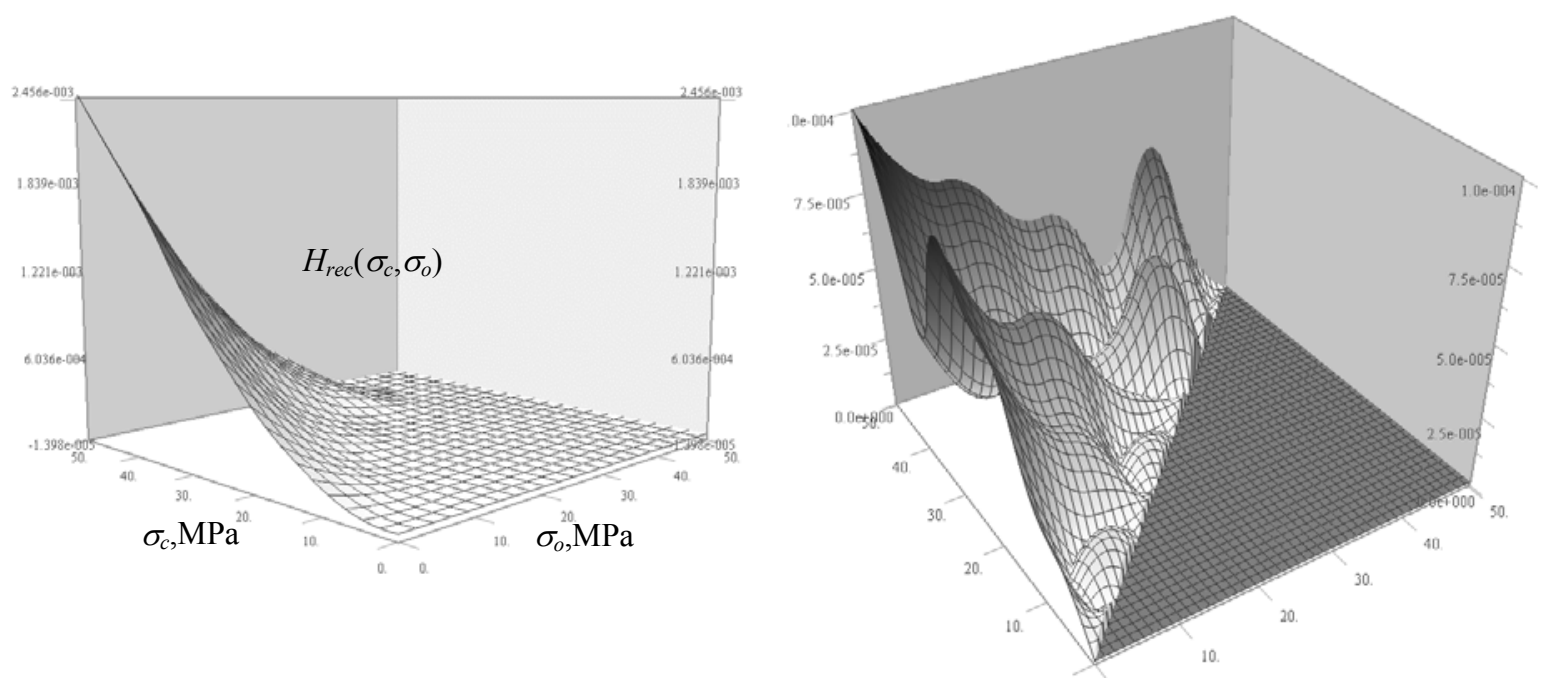

Figure 6 . The reconstructed IPM density $H_{r e c}\left(\sigma_{c}, \sigma_{o}\right)$, left, and the local reconstruction error, right, for synthetic data contaminated with additive noise $\left(\eta=3 \cdot 10^{-5}\right)$ based on the PM density given by Eq.(14) for $d_{\perp}=30 \mathrm{MPa}$ and $d_{\|}=50 \mathrm{MPa}$. The global reconstruction error is $3 \%$ for a regularization parameter $\alpha=0.5$, a $3^{\text {rd }}$ degree polynomial approximation of the smallest loops and a $6^{\text {th }}$ degree approximation for the others loops. Splines of the $3^{\text {rd }}$ order were used to retrieve the entire distribution function from the discrete IPM grid data. 


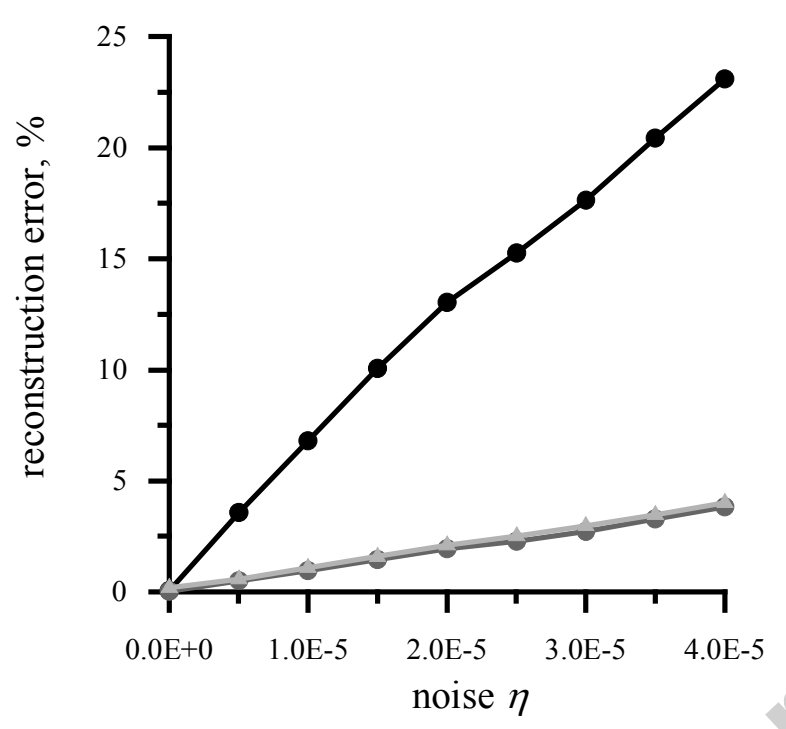

Figure 7. Global reconstruction errors for different noise amplitudes $\eta$ using the optimized parameters of the algorithm. Black: global reconstruction error on the bin integrals, dark gray: global reconstruction error on the gridded IPM density, light gray: global reconstruction error on the spline approximated IPM density. 


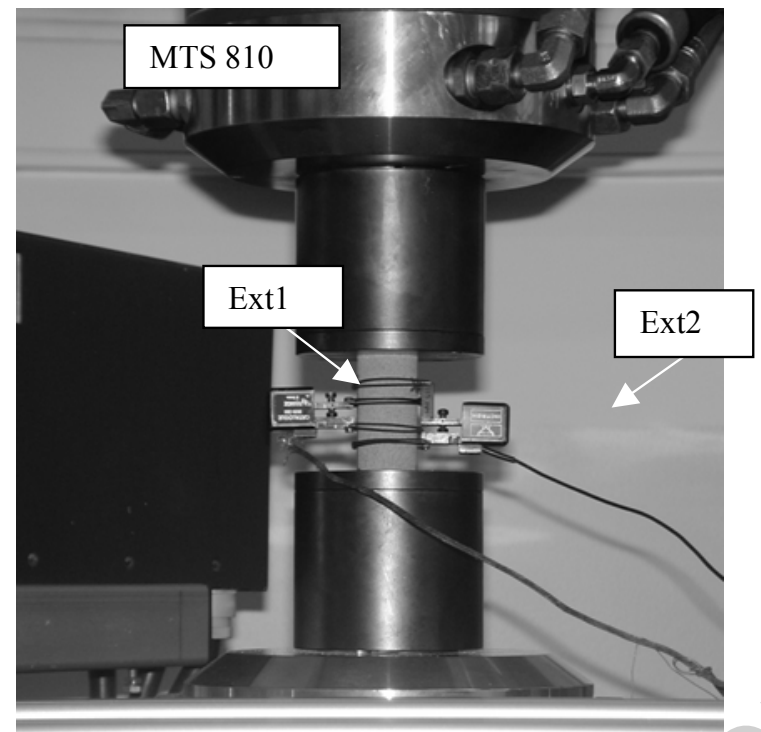

Figure 8. Experimental set-up for cyclic uniaxial compression tests on cylindrical rock samples. 


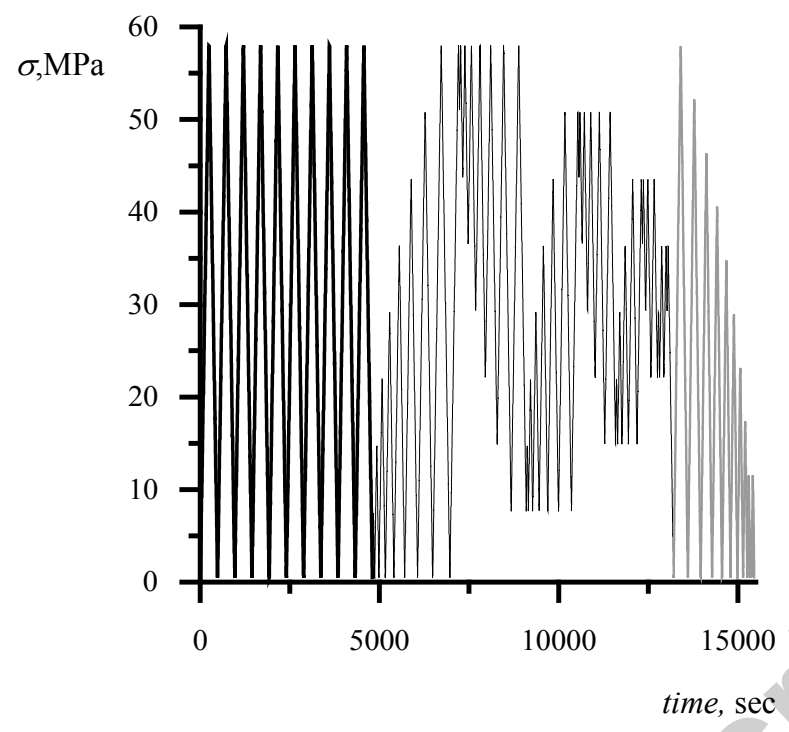

Figure 9. Typical experimental stress command protocol, including initialization cycles (time $<5000 \mathrm{sec}), 8$-level stress protocol for reconstruction analysis $(5000<$ time $<$ $13000 \mathrm{sec}$ ), and additional cycling for validating the reconstruction (time $>1300 \mathrm{sec}$ ). 


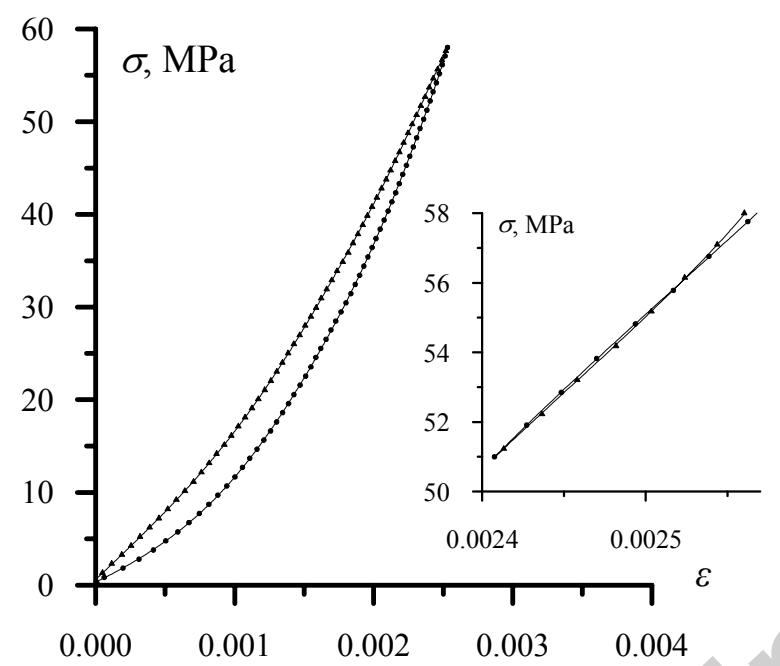

Figure 10. Experimental data corresponding to the largest loop of the 8-level stress protocol $(0 \rightarrow 58 \mathrm{MPa} \rightarrow 0)$ and of one of the smallest loops $(58 \mathrm{MPa} \rightarrow 51 \mathrm{MPa} \rightarrow 58 \mathrm{MPa})$ for a sample of Serena sandstone (each $20^{\text {th }}$ point is plotted). The solid lines represent polynomial approximation of $3^{\text {rd }}$ and $6^{\text {th }}$ degree for the small and the large loop, respectively. 


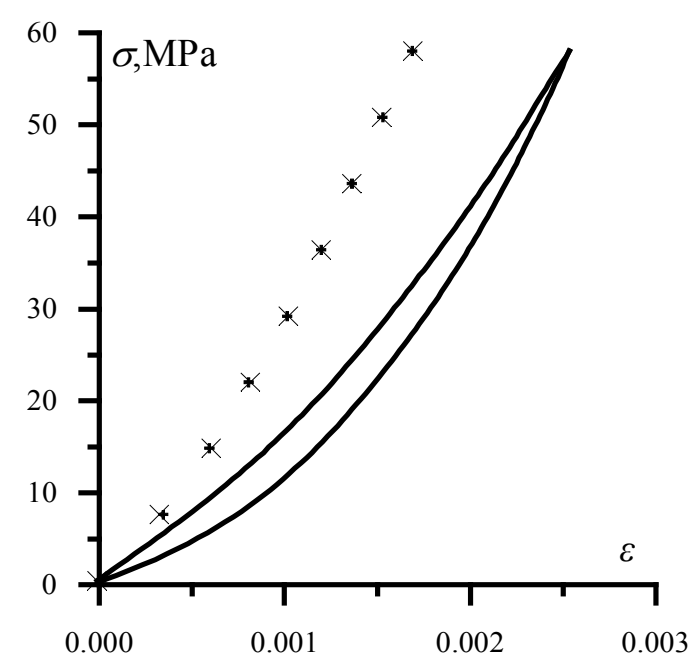

Figure 11. Non-hysteretic contribution $\varepsilon_{N H}(\sigma)$ (discrete data points) and the largest hysteretic loop (solid lines) in the stress-strain behavior of Serena Sandstone. The small crosses correspond to the inversion of $\varepsilon_{N H}(\sigma)$ from the loading curve (Eq.12) and the large ones are obtained from the unloading curve (Eq. 13). 

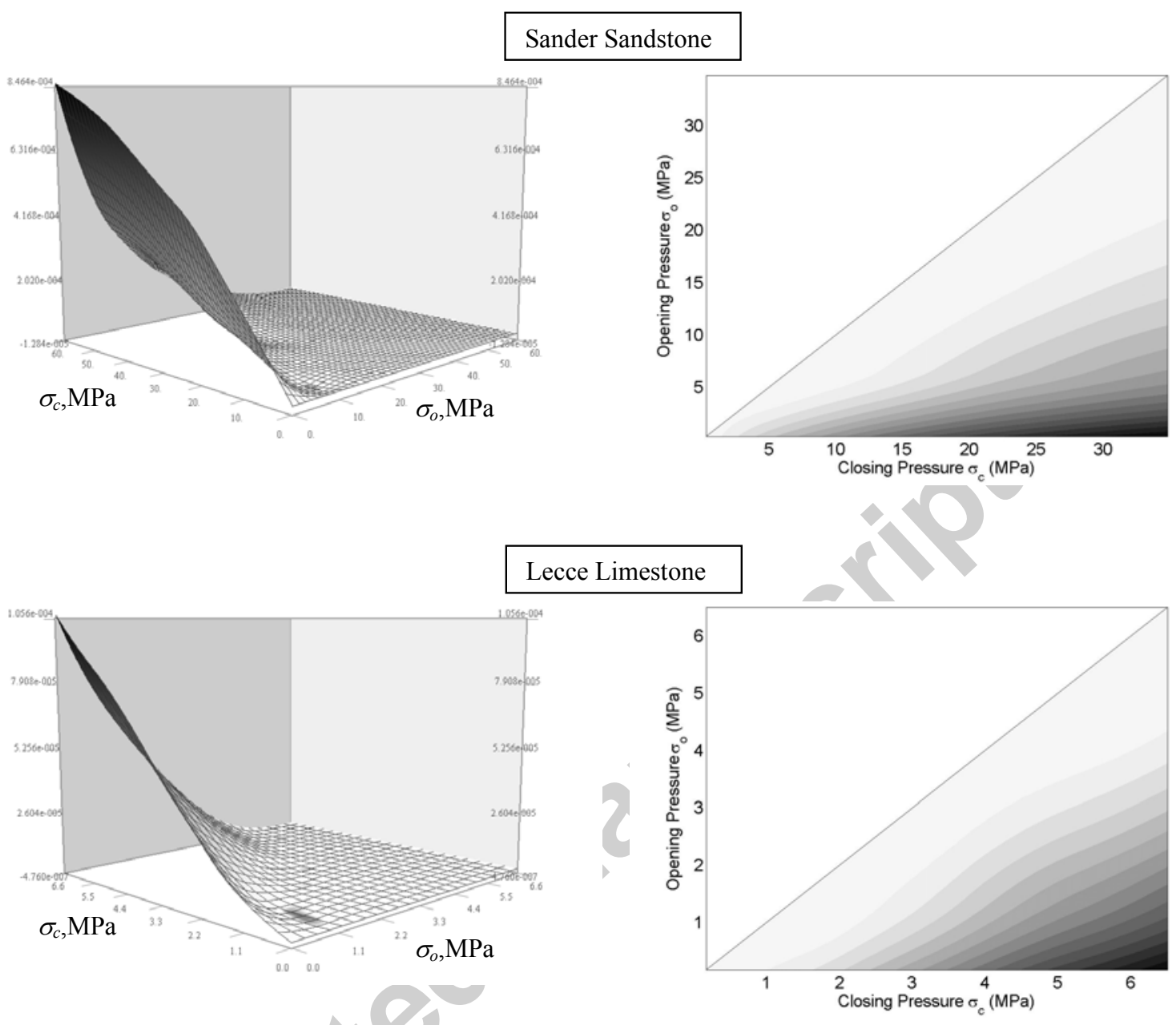

Figure 12. Reconstructed IPM density distributions (left in 3D, right in contourplot) for two different rocks (Serena sandstone and Lecce limestone). 

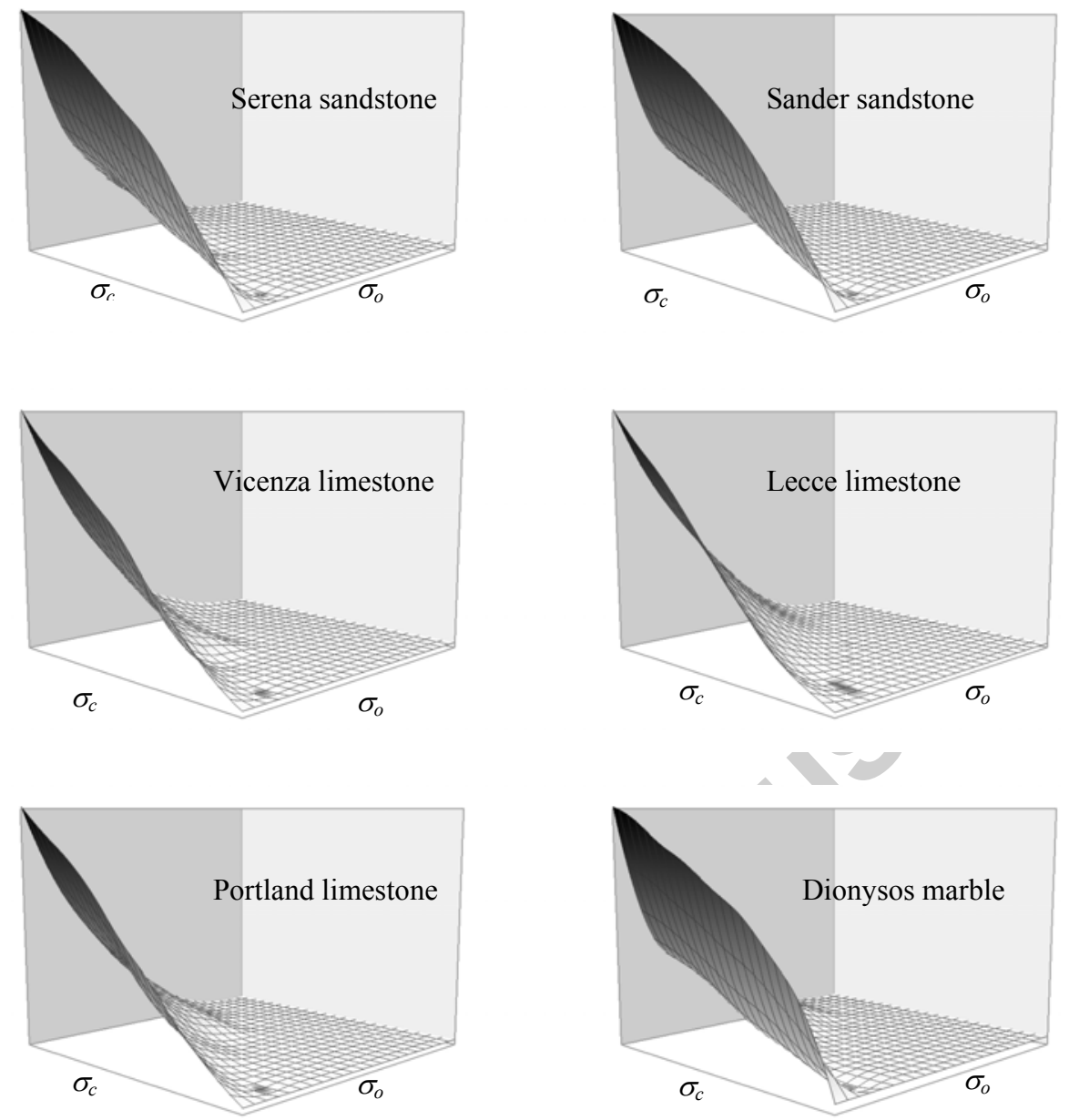

Figure 13. Reconstructed IPM portraits for various rocks. In the right column: Serena and Sander sandstone. In the right column: Portland limestone, Vicenza limestone and Lecce limestone. The values $\sigma_{c}$ and $\sigma_{o}$ range from 0 to $\sigma_{60 \%}(60 \%$ of UCS) for each rock. The maximum IPM density $H_{\max }$ corresponding to this interval is listed in Table 3 and defines the color scale of the plot. 

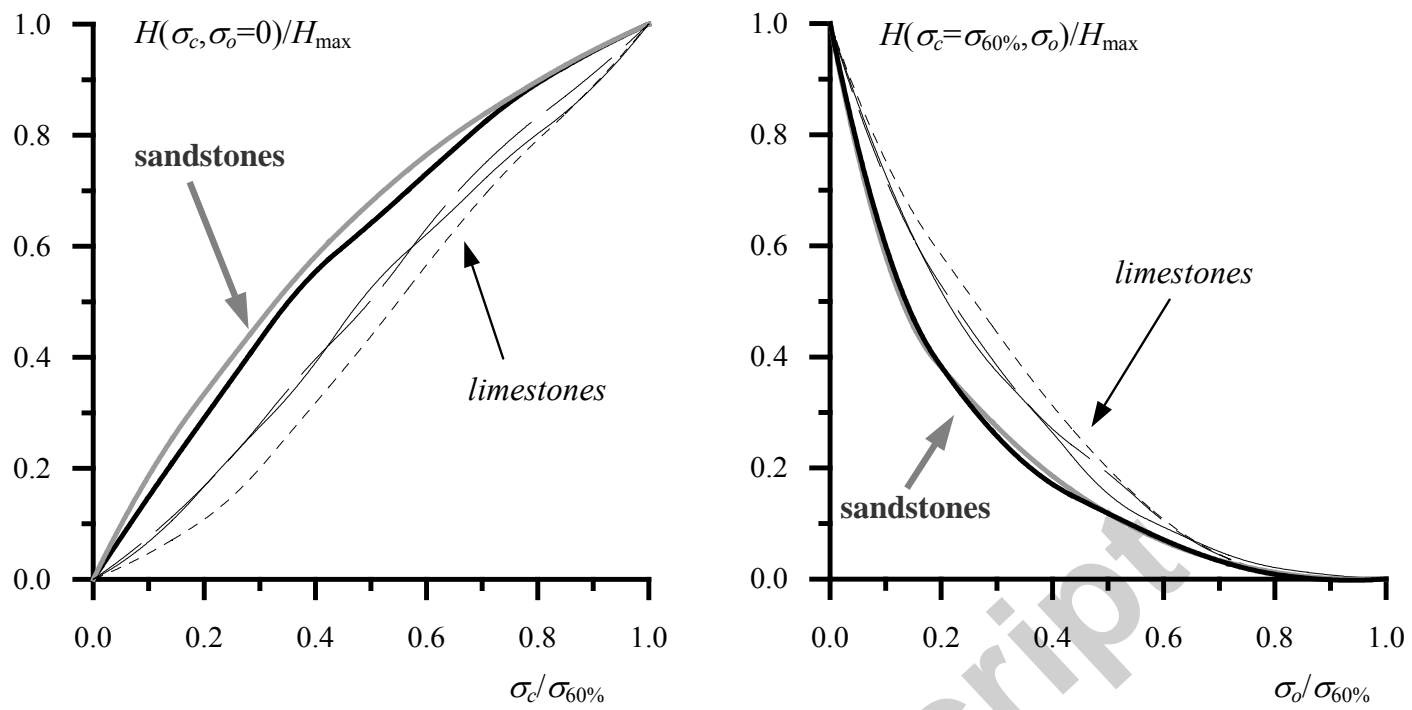

Figure 14. Comparison of the normalized IPM densities at $\sigma_{o}=0$ and at $\sigma_{c}=\sigma_{60 \%}$ for different rocks. The thick black line represents Serena sandstone, the thick gray line is for Sander sandstone, the thin black line is for Vicenza limestone, the dotted line is for Lecce limestone, and the dashed line is for Portland limestone. 


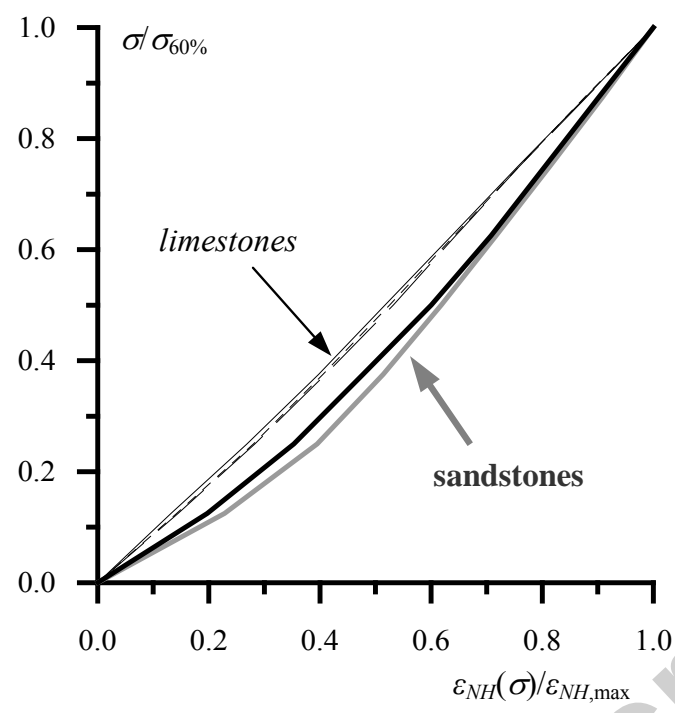

Figure 15. Comparison of the normalized non-hysteretic contribution for different rocks. Same line-style as in Figure 14. 


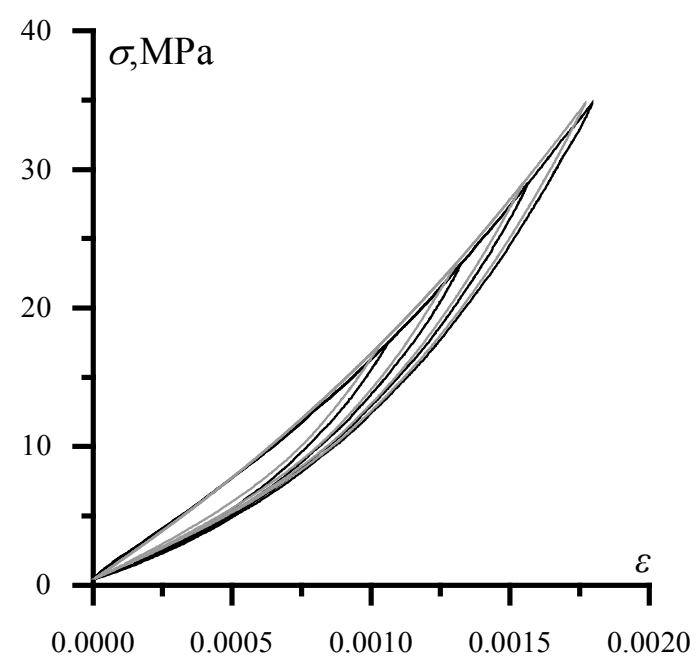

Figure 16. Prediction of additional loading-unloading cycles made for Sander sandstone. Black - actual experimental data; Gray - prediction based on the reconstructed IPM density. The relative RMS of the deviation between prediction and actual measurement is $2 \%$. 